\title{
ASPECTOS NUTRICIONAIS EM IDOSOS
}

\section{Ana Luiza Rios Antunes'; Munah Najeh Saleh Ahmad Maruf2; Flávia Dorneles Saleh $^{3}$; Luana Dall Asta de Almeida ${ }^{4}$; Luiza Fortes Lamberty ${ }^{5}$; Eduarda Rodrigues Machado ${ }^{6}$; Bianca Vieira Baldin ${ }^{7}$; Maria Helena Gehlen ${ }^{8}$}

\section{RESUMO}

Introdução: A avaliação nutricional é um processo que vem crescendo a cada dia no Brasil, tendo em vista que é um país em transformação. Por mais que esteja sendo difícil de transmitir a todas as regiões, vem ganhando força e conseguindo levar à prevenção, promoção e recuperação em saúde. Metodologia: É um estudo de natureza teórico-reflexivo, cuja fundamentação baseia-se na formulação discursiva acerca da temática. Consultado em materiais acadêmicos científicos, onde foram relativos aos aspectos nutricionais em idosos. Resultados: O processo de senescência traz junto de si uma diminuição do processo metabólico, trazendo então a importância de proporcionar uma boa introdução alimentar e educação em saúde aos idosos. Conclusão: Conclui-se que é de suma relevância que a equipe de referência estimule a prática de exercícios físicos e oficinas comunitárias. Incluindo atividades que desenvolvam técnicas de movimentos e educação alimentar, criando hortas comunitárias para que todos possam usufruir.

Palavras-chave: Nutrição; Longevos; Saúde.

\section{ABSTRACT}

\footnotetext{
${ }^{1}$ Acadêmica do curso de Enfermagem. Universidade Franciscana UFN. Email: riosana1306@gmail.com

${ }^{2}$ Acadêmica do curso de Enfermagem. Universidade Franciscana UFN. Email: munahsaleh1999@gmail.com

${ }^{3}$ Acadêmica do curso de Enfermagem. Universidade Franciscana UFN. Email: flaviasaleh222@gmail.com

${ }^{4}$ Acadêmica do curso de Enfermagem. Universidade Franciscana UFN. Email: luanadallmeida@gmail.com

${ }^{5}$ Acadêmica do curso de Enfermagem. Universidade Franciscana UFN. Email: luizaflamberty@gmail.com

${ }^{6}$ Acadêmica do curso de Enfermagem. Universidade Franciscana UFN. Email: eduardamachado886@gmail.com

${ }^{7}$ Enfermeira graduada pela Universidade Franciscana. Email: biancavbaldin@gmail.com

${ }^{8}$ Professora supervisora da disciplina. Universidade Franciscana UFN. Email: gehlenmh@gmail.com
} 
Objective: Nutritional assessment is a process that has been growing every day in Brazil, considering that it is a country in transformation. As difficult as it is to all regions, it has been gaining strength and managing to lead to prevention, promotion and health recovery. Methodology: It is a theorical-reflective study, whose foundation is based on the discursive formulation about the theme. Consulted in scientific academic materials, where they were related to nutritional aspects in the elderly. Results: The senescence process brings whith it a decrease in the metabolic process, thus bringing the importance of providing a good introduction to food and health education for the elderly. Conclusion: It is concluded tha it is extremely important the reference team encourages the practice of physical exercises an community workshops. Including activities that develop movement techniques and food education, creating community gardens for everyone to enjoy.

Key words: Nutrition; Elderly; Health.

Eixo Temático: Atenção Integral e Promoção à Saúde (AIPS)

\section{INTRODUÇÃO}

Atualmente sabe-se que o Brasil passa por uma transformação, na qual a população está gradativamente envelhecendo. Com isso, medidas de prevenção e promoção à saúde vêm sendo ampliadas e efetuadas, para que possamos ter um aumento na taxa de expectativa e qualidade de vida aos longevos. Porém, vê-se uma certa dificuldade pelo fato de estas transformações serem desiguais entre as regiões brasileiras por conta de doenças crônicas e agudas, o que torna este processo um pouco mais lento e demorado (TAVARES et al, 2015).

As avaliações nutricionais e de alimentação estão tornando-se cada vez mais presentes na Atenção Integral à Saúde do Idoso e na Política Nacional de Saúde da Pessoa Idosa. Junto, traz também a valorização da humanização e Determinantes Sociais da Saúde, para então assegurar que todos tenham um envelhecimento digno, respeitado e satisfatório (TAVARES et al, 2015).

A população idosa está inserida em um contexto de risco que possui maior probabilidade de desnutrição, visto que, dificilmente possuem hábitos de atividades físicas regular. Outros fatores que colaboram para este aspecto é a falta de alimentação balanceada, desregulação do sono, tabagismo, condições socioeconômicas e doenças crônicas. Com base nestas evidências, torna-se nítida a necessidade de ter uma visão voltada aos idosos para ajudar a melhorar estes aspectos (TAVARES et al, 2015). 
A blue zone são áreas azuis do Brasil, onde vivem longevos menores e maiores de 100 anos de idade. Que por sua vez apresentam grande qualidade de vida e saúde, não só física como mental. Trata-se de uma zona de grande referência para a população idosa, pois mostra que é possível ser idoso, saudável, feliz, garantir e prolongar sua autonomia (BUETTNER, 2019).

\section{METODOLOGIA}

Este respectivo estudo é de natureza teórico-reflexivo, cuja fundamentação baseia-se na formulação discursiva acerca da temática. Consultado em materiais acadêmicos científicos, onde foram relativos aos aspectos nutricionais em idosos. Mostrando e evidenciando aspectos importantes e relevantes ao longevo deste processo.

\section{RESULTADOS E DISCUSSÕES}

Podemos afirmar que, além dos que citaremos abaixo, um dos pontos norteadores para estabelecer um plano de cuidados ao longevo é a história prévia de hábitos alimentares presentes ao longo da vida. Tendo em vista que o organismo mostrará os resultados das ingestões alimentares e estilo de vida durante a longevidade. Devemos levar em consideração neste plano de cuidados que as necessidades nutricionais dos idosos são restritas ao processo do envelhecimento (SANTOS; MACHADO; LEITE, 2010).

Ainda não existe um padrão de utilização global instrumental para o acompanhamento e diagnóstico da disfunção nutricional em idosos, de tal forma que nos leva a atentar para um cuidado redobrado na escolha da melhor ferramenta para a questão. O uso do IMC calcula o peso "ideal" em relação à altura, sendo um dos mais utilizados, porém se não aliado a outras ferramentas como a bioimpedância que avalia a porcentagem de gordura e água presentes no organismo, não pode ser considerado fidedigno (SANTOS; MACHADO; LEITE, 2010).

Ademais, a avaliação bioquímica pode revelar através de exames laboratoriais, deficiências de nutrientes e outras enzimas presentes na corrente sanguínea, assim como os processos de quebra e síntese de moléculas que podem levar a perda ou 
diminuição das funções corpóreas, como por exemplo o processo catabólico e anabólico, onde há uma maior quebra de moléculas e menor produção das mesmas no organismo, o qual se apresenta no processo da senescência. (TRAMONTINO, et al, 2009).

Dentro do estudo antropométrico, além do IMC se encontram diversas estruturas individuais que quando unidas formam um método eficiente e funcional no diagnóstico e prognóstico da avaliação nutricional do cliente. Este conjunto de ferramentas, aliado a uma anamnese bem conduzida proporciona um diagnóstico mais completo e qualificado. Portanto, os idosos em processo de longevidade, necessitam de acompanhamento e avaliação da equipe de saúde, sendo inegável o processo interdisciplinar para que o cuidado seja além de humanizado, efetivo (CLARES; FREITAS, 2013).

A enfermagem se estabelece imprescindível no cuidado aos mesmos, pois muitas vezes está à frente dos atendimentos na atenção primária. Se responsabiliza pela educação, promoção, prevenção, recuperação e manutenção da saúde, proporcionando assim, qualidade e dignidade de vida durante esse processo natural e fisiológico do ser humano (CLARES; FREITAS, 2013).

É de suma importância atentar para a nutrição, desde a infância até o envelhecimento, visto que, ela reflete em todas as faixas etárias do ciclo da vida. Ter uma boa alimentação e realizar a prática de atividades físicas é um fator essencial para poder obter uma boa qualidade de vida durante está nova. Deve-se considerar às mudanças metabólicas, na qual torna-se mais lenta com o passar dos anos, buscando alimentos leves e saudáveis para facilitar no processo (CLARES; FREITAS, 2013)

É necessário para a equipe de referência em saúde transmitir sempre o máximo possível de conhecimento e informações sobre alimentos necessários e importantes para manter um bom balanceamento nutricional. Junto às informações, oferecer formas e métodos socioeconômicos compatíveis com a renda de cada longevo (CLARES; FREITAS, 2013). 


\section{CONCLUSÃO}

Portanto, conclui-se que é de grande importância e relevância transmitir a prevenção e promoção de saúde aos idosos em ambientes de saúde e campanhas governamentais com relação a alimentos e a importância da nutrição. Para assim então, obtermos pessoas longevas, saudáveis e autônomas.

É de grande valia estimulá-los a prática de exercícios e atividades físicas, a obter uma alimentação mais balanceada, saudável e práticas que auxiliam na melhora de sua autoestima e autonomia. Nota-se que seria de grande relevância a criação de hortas, visto que auxilia na habilidade, técnica, a manter uma alimentação orgânica e saudável para os longevos que não possuem condições econômicas para então poder se alimentar melhor.

A realização de oficinas nas comunidades também seria eficaz, trazendo atividades como plantações, aulas e rodas de conversas sobre nutrição e alimentos saudáveis, aulas de dança, ginástica e outras atividades que a população desejar.

\section{REFERÊNCIAS}

BUETTNER, Dan. As zonas azuis da felicidade: Lições das Pessoas Mais Felizes do Planeta. ed 1. São Paulo: nVersos, 2019.

CLARES, Jorge Wilker Bezerra; FREITAS, Maria Célia. Diagnósticos de Enfermagem do domínio Nutrição identificados em idosos da comunidade. Rev. Eletrônica de Enfermagem. Ceará, 2010. Disponível em: https://revistas.ufg.br/fen/article/view/20513. Acesso em 11 de junho de 2020.

SANTOS, Ana Célia Oliveira; MACHADO, Myrtes Maria de Oliveira; LEITE, Elder Machado. Envelhecimento e alterações do estado nutricional. Rev.Inst. Ciê. Bio. v. 21, n. 2, p. 169-210. Pernambuco, 2010. Disponível em: http://ggaging.com/details/274/pt-BR. Acesso em 11 de junho de 2020.

TAVARES, Elda Lima et al. Avaliação nutricional de idosos: desafios da atualidade. Rev. bras. geriatr. gerontol. Rio de Janeiro, v. 18, n. 3, p. 643-650, 2015. 
EDUCACÃO, SAÚDE

ETECNỎLGIA

26 A 28 DE OUTUBRO DE 2021

\section{QUFN}

Disponível em: https://www.scielo.br/scielo.php?script=sci arttext\&pid=S1809$\underline{98232015000300643}$

TRAMONTINO, Vanessa Silva et al. Nutrição para idosos.Rev. de Odontologia da Univ. Cid de são Paulo. v. 12, n. 2, p. 102-300. São Paulo, 2009. Disponível em: http://publicacoes.unicid.edu.br/index.php/revistadaodontologia/article/view/465/359. Acesso em 11 de junho de 2020. 